\title{
Cryogenic STEM Imaging and Spectroscopy of Electron Beam Sensitive Materials
}

Katherine A. Spoth ${ }^{1}$, Michael J. Zachman ${ }^{1,2}$, David A. Muller ${ }^{1,3}$ and Lena F. Kourkoutis ${ }^{1,3^{*}}$

1. School of Applied and Engineering Physics, Cornell University, Ithaca, NY, USA.

2. Present address: Center for Nanophase Materials Sciences, ORNL, Oak Ridge, TN, USA.

${ }^{3 .}$ Kavli Institute at Cornell for Nanoscale Science, Cornell University, Ithaca, NY, USA.

*Corresponding author: lena.f.kourkoutis@cornell.edu

Recent advances in cryogenic electron microscopy have enabled high-resolution characterization of materials that are incompatible with imaging conditions typically used in the physical sciences. A range of reactive materials, soft/hard hybrid systems as well as liquid/solid interfaces all directly benefit from cryogenic sample cooling. It allows liquids to be preserved through vitrification and chemically reactive samples to be studied by significantly reducing rates of reactions with the environment. Cryo-S/TEM has, therefore, enabled atomic-resolution imaging and nanoscale chemical mapping of reactive and beamsensitive materials, such as lithium in rechargeable batteries [1-3]. Cryogenic temperatures also reduce the critical dose for damage observed in materials. At liquid nitrogen temperature, sample cooling has shown to raise the critical dose of many materials by a factor of 2-3 and by up to 100 for some [4]. Atomic motion and diffusion rates in the sample are reduced at low temperature, likely resulting in fewer broken bonds by radiolysis and reduced atom movement after the bonds are broken thereby preventing mass loss.

The critical dose in a single material depends, however, on the specific structural or electronic feature that is probed. Focusing on vitrified organic electrolytes used in batteries, we recorded a series of spectroscopic maps to analyze the critical doses at which different types of damage occurs. Small bubbles appear rapidly, beginning with the first cryo-STEM image recorded and at doses below $10 \mathrm{e}^{-} / \AA^{2}$. These bubbles are most likely due to hydrogen liberated from the electrolyte solvent molecules [4]. For substantial mass loss and modification of spectral fine structure, however, doses greater than $10^{3} \mathrm{e}^{-} / \AA^{2}$ are required. The carbonate portion of the solvent molecules was most stable, and the EELS fine structure associated with this part of the molecule survives even at high dose [3]. For lithium compounds, a dose one order of magnitude higher than that for organic electrolytes is acceptable for accurate characterization of bonding states (Fig. 1).

While cryo-STEM/EELS enables nanoscale chemical mapping of beam sensitive materials (Fig. 2), resolution is still limited by damage. A promising approach to STEM imaging of electron beam sensitive materials is to combine cryo-cooling with the use of pixelated direct detectors for STEM. Here, we make use of the electron microscope pixel array detector (EMPAD) to collect the full diffraction pattern at each scan pixel comprising nearly all of the electrons incident on the specimen [5]. Such detectors create new possibilities for STEM imaging with higher dose-efficiency as they allow flexible choice of scattering angles as well as new types of imaging modes not possible with a traditional setup. One such technique allows us to form a coherent image using all the electrons in the bright field (BF) disk called tilt-corrected BF STEM (tcBF-STEM). The EMPAD acts as an array of coherent detectors - each pixel is small compared to the convergence angle of the beam and those located within the BF disk each produce a phase-contrast image. By carefully combining the images produced by each pixel in the BF disk, we obtain a coherent image that utilizes significantly more of the incident beam than a conventional BF image. We demonstrate tcBF-STEM on thin and thick hydrated frozen biological specimen (Fig. 3). For thick, whole E. coli cells significantly improved information transfer is observed compared to typically used EFTEM imaging. In thinner specimens such as ribosomes embedded in vitreous ice a larger fraction of electrons 
is scattered into the BF region. The additional signal should allow similar quality imaging with smaller incident dose, important for preserving high-resolution structural information [6].

References:

[1] Y Li et al., Science 358 (2017), p. 506.

[2] X Wang et al., Nano Lett. 17 (2017), p. 7606.

[3] MJ Zachman et al., Nature 560 (2018), p. 345.

[4] RF Egerton, P Li and M Malac, Micron 35 (2004), p. 399.

[5] MW Tate et al., Microscopy and Microanalysis 22 (2016), p. 237.

[6] Supported by NSF (DMR-1654596, DMR-1429155, DMR-1719875) and the Packard Foundation.
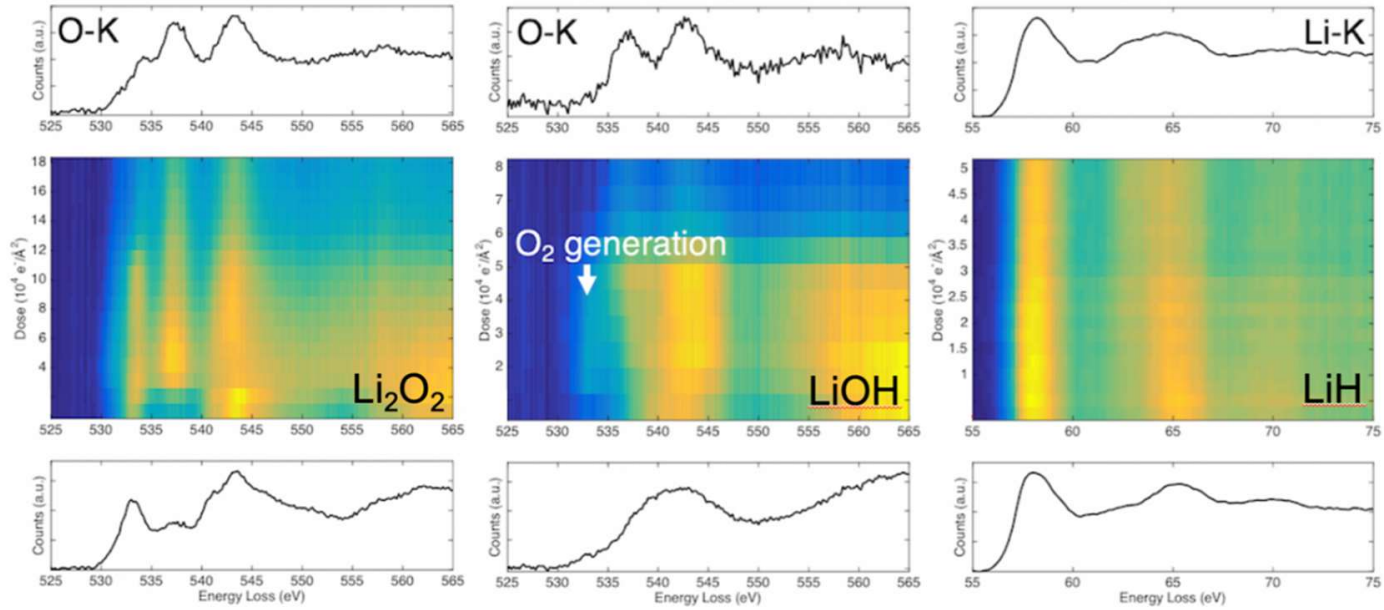

Figure 1. Electron beam induced EELS fine structure changes of lithium compounds measured at cryogenic temperatures. While lithium oxides convert to $\mathrm{Li}_{2} \mathrm{O}$ under large doses, $\mathrm{LiH}$ is primarily affected by mass loss, with no substantial changes in the Li-K edge fine structure [3].
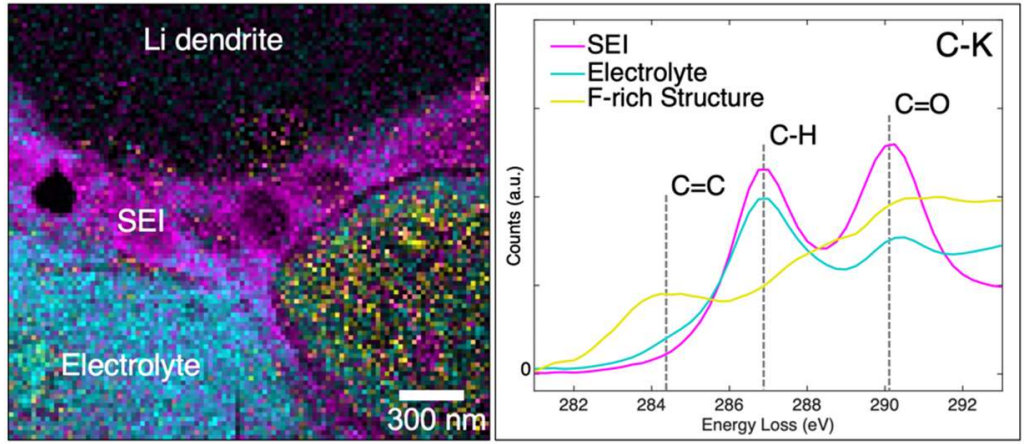

Figure 2. Operating well below the critical dose for radiation damage which affect the C-K edge fine structure, cryoSTEM/EELS allows mapping of the $\mathrm{C}$ bonding environments at an electrolyteelectrode interface. The increased $\mathrm{C}=\mathrm{O}$ peak is consistent with a lithium ethylene dicarbonate SEI layer. Ethylene gas produced during SEI formation may explain the bubbles seen on the left [3].
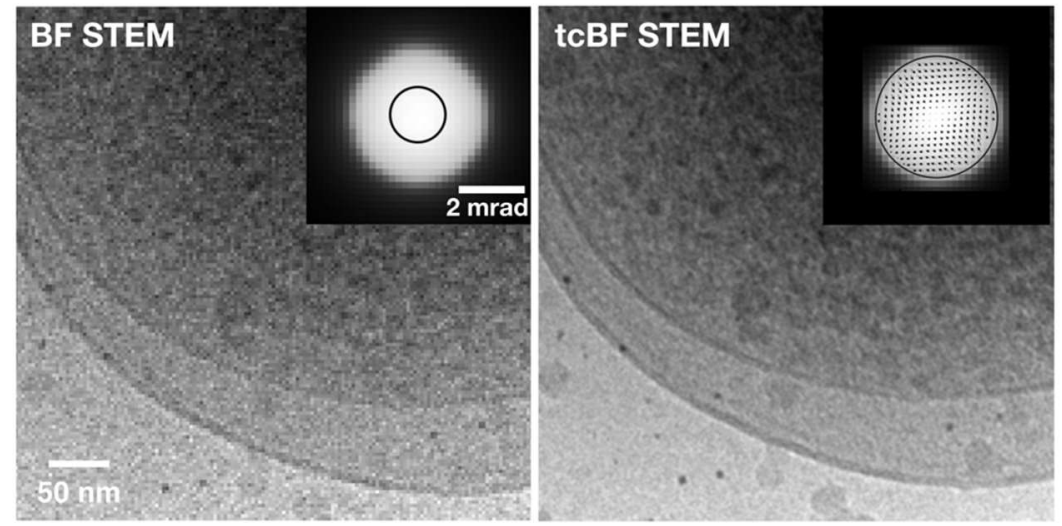

Figure 3. Dose-efficient cryo-STEM performed on a frozen hydrated specimen using the EMPAD. A CBED pattern is collected at each scan position. (left) Bright field image formed with a conventional STEM detector geometry. (right) The tcBF-STEM image is the sum of cross-correlated images formed from each pixel in the central disk of the CBED pattern, using 5 times more of the

incident dose than the conventional BF-STEM image. 